\title{
INDICADORES DE TRANSPORTE E MOBILIDADE URBANA PARA AS CIDADES SAUDÁVEIS - CASO DA ÁREA METROPOLITANA DE LISBOA, PORTUGAL TRANSPORT AND URBAN MOBILITY INDICATORS FOR HEALTHY CITIES - CASE STUDY OF LISBON METROPOLITAN AREA, PORTUGAL
}

\author{
Ana Louro ${ }^{1}$, Nuno Marques da Costa ${ }^{1}$ \\ ${ }^{1}$ Universidade de Lisboa (UL), Lisboa, Portugal
}

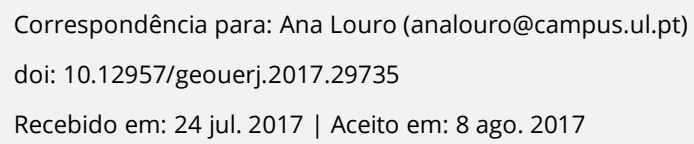

\section{RESUMO}

O conceito de Cidade Saudável desenvolvido pela Organização Mundial da Saúde é um conceito holístico que abarca os distintos determinantes da saúde repartidos pelos domínios social, económico e ambiental. Neste sentido, a organização do transporte, que permite o acesso ao emprego, bens e serviços, mas, simultaneamente, polui, consome recursos naturais e é causador de sinistralidade, constitui um elemento de extrema relevância para a concretização dos princípios da cidade saudável. Neste artigo propõe-se a definição de um sistema de indicadores que permita avaliar a contribuição do transporte para a concretização da cidade saudável, recorrendo a dados disponíveis nas fontes do sistema estatístico português. O sistema de indicadores será aplicado à Área Metropolitana de Lisboa (Portugal), sendo as unidades de análise os seus municípios.

Palavras-chave: transporte; mobilidade urbana; cidades saudáveis; indicadores; Área Metropolitana de Lisboa.

\section{ABSTRACT}

The concept of Healthy City developed by the World Health Organization is a holistic concept which covers all determinants of health, divided by social, economic and environmental domains. In this sense, transport organization, allowing the access to workplaces, goods and services, but at the same time, is source of pollution, natural resource consuming and road accidents, elements of extreme relevance to the implementation of healthy city principles. In this paper, it is proposed the definition of an indicator system to evaluate the contribution of transport to healthy cities, recurring to available indicators in the Portuguese statistical system. This indicator system will be applied to Lisbon Metropolitan Area (Portugal), having its municipalities as units of analysis.

Keywords: transport; urban mobility; healthy cities; indicators; Lisbon Metropolitan Area.

\section{INTRODUÇÃO}

A abordagem à saúde evoluiu no tempo tendo passado de uma perspetiva focada na doença, ou ausência de saúde, para uma perspetiva holística onde ao estado de saúde se associam fatores biológicos, psicológicos, sociais e ambientais que determinam a saúde do indivíduo (TORRES et al., 2013). Dooris (1999) assume que a saúde se posiciona no centro do Desenvolvimento Sustentável, onde se cruzam as dimensões social, ambiental e económica. É neste contexto que emerge o conceito de “cidade saudável” associado a territórios e comunidades, hoje essencialmente urbanos (cerca de 54\% da população mundial e 75\% da população da União Europeia vive em áreas urbanas (AEA, 2006; 
UE, 2016; UN, 2016)). Igualmente, no centro da discussão do Desenvolvimento Sustentável encontra-se a temática do transporte e mobilidade urbana (BANISTER, 2005; RODRIGUE et al., 2006), sendo que as tendências do consumo de transporte revelam-se divergentes ao objetivo de sustentabilidade, seja pelo crescente consumo de combustíveis fósseis e pela emissão de poluentes, seja pelo aumento da sinistralidade rodoviária (BANISTER, 2005).

Este trabalho apresenta dois objetivos: 1) identificar indicadores do sistema estatístico português que representem o contributo do domínio "transporte e mobilidade urbana” para a cidade saudável, e 2) analisar esses indicadores considerando os municípios da Área Metropolitana de Lisboa de forma a identificar as principais desigualdades. Esta sistematização será útil para os trabalhos da Rede Portuguesa de Municípios Saudáveis, bem como para departamentos de transporte e mobilidade das autarquias locais ou entidades metropolitanas (AML ou Instituto da Mobilidade e Transportes).

O artigo organiza-se em cinco partes. Na primeira parte é apresentado o conceito de cidade saudável e a sua relação com o domínio "transporte e mobilidade urbana". A segunda parte contextualiza a AML como área de estudo. A terceira parte apresenta a metodologia aplicada com destaque para a seleção dos indicadores disponíveis no sistema estatístico português que permitem responder à discussão concetual anterior. Na quarta parte analisam-se os indicadores elegidos para os municípios da AML, sublinhando-se as principais desigualdades. A quinta e última parte apresentará as considerações finais.

\section{O PAPEL DO TRANSPORTE E MOBILIDADE URBANA PARA AS CIDADES SAUDÁVEIS}

Apesar dos benefícios que a urbanização trouxe para as populações, alguns impactes negativos na saúde das comunidades urbanas podem ser assinalados resultantes de problemas na qualidade da água e qualidade alimentar, falta de gestão dos resíduos urbanos, poluição atmosférica, contaminação de solos ou qualidade da habitação, entre outros. No entanto, as contínuas reformas sanitárias e o desenvolvimento da medicina, têm contribuido de forma decisiva para a melhoraria das condições de saúde da população (VLAHOV, 2004; WILLIAMS, 2013). 
Para a Organização Mundial de Saúde (OMS), a cidade saudável fundamenta-se num processo de consciência da saúde e não apenas no objetivo de atingir um nível de saúde pré-definido (WHOEUROPE (1), n.d.). Hancock \& Duhl (1988, p.24) apresentaram uma das primeiras definições de cidade saudável como "uma cidade que está continuamente a criar e melhorar os ambientes físicos e sociais e a expandir os recursos comunitários que permite às pessoas apoiarem-se mutuamente na execução de todas as funções da vida e desenvolver o seu potencial máximo". Este conceito abrange os fatores que influenciam a saúde das pessoas e das comunidades de forma múltipla e interativa (CENTRE FOR HEALTH PROTECTION, 2009), ou seja, considera "as circunstâncias em que as pessoas nascem, crescem, vivem, trabalham e envelhecem" (WHO-EUROPE (2), n.d.), sendo a esquematização dos determinantes da Saúde de Whitehead \& Dahlgren (1991) um dos mais representativos.

Considerando-se “(...) a saúde (como) um direito humano básico e (...) essencial ao desenvolvimento social e económico" (WHO, 1997), esta tornou-se fulcral no contexto das políticas europeias desde a década de 1970, com a Conferência das Nações Unidas sobre o Meio Ambiente Humano (1972) e a 30ª Assembleia da Organização Mundial de Saúde (1977). Na década seguinte, do Relatório de Brundtland (1987) emergiu o conceito de Desenvolvimento Sustentável. Simultaneamente surgiu o Projeto Cidades Saudáveis (PCS), resultado da Carta de Ottawa para a Promoção da Saúde (1986) que, por sua vez, se ancorou no documento "As metas da saúde para todos" da OMS (1977), na Declaração sobre os Cuidados de Saúde Primários de Alma-Ata (1978) e na 34ª Assembleia Mundial de Saúde (1981). Assim, o Projeto Cidades Saudáveis é associado a estratégias para o desenvolvimento e implementação da promoção da saúde, conjugando a teoria e a prática da saúde pública, nunca esquecendo a sua contribuição para o Desenvolvimento Sustentável (DOORIS, 1999).

A Rede Europeia de Cidades Saudáveis (RECS) conta atualmente com cerca de 1000 cidades e vilas de 30 países, tendo como objetivo principal colocar a saúde nas agendas social, económica e política dos governos locais, suportado nos valores da equidade, governança participativa e solidária, colaboração intersetorial e ação sobre os determinantes de saúde (WHO-EUROPE (2), n.d.). 
Em Portugal, a Rede Portuguesa de Municípios Saudáveis (RPMS) surgiu em 1997 como associação de municípios, ficando responsável por divulgar, implementar e desenvolver o PCS, contando atualmente com 45 municípios associados, 13 deles na AML. No contexto português, o conceito de “cidade saudável" é convertido em "município saudável” por um motivo; embora o Movimento Cidade Saudável tenha a cidade como unidade territorial preferencial, no caso português a intervenção centrase no município, justificada pela atuação autárquica não dirigida apenas às cidades ou áreas urbanas, mas a todo o seu território e população, tal como enunciado pela Rede Portuguesa de Municípios Saudáveis. No contexto da ação dos municípios da RPMS, LOURO et al. (2017) referem a mobilidade urbana como uma das áreas de intervenção dos PCS, onde, nomeadamente, se identificam intervenções associadas à segurança rodoviária, em particular orientadas para as crianças em idade escolar.

São várias as formas como o transporte e a mobilidade urbana promovem a cidade saudável, sendo fundamental reconhecer as diferentes necessidades dos indivíduos neste domínio de forma a criar uma cidade inclusiva (ALVES et al., 2017). De acordo com Mindell et al (2011, em FREUDENBERG \& GALEA, 2011, 84), “os ganhos do transporte sustentável e da saúde reforçam-se mutuamente, (...) contudo, devem ser considerados no contexto um do outro para garantir que os resultados interessem aos objetivos de ambas as áreas". Davis (em TOLLEY, 2003) refere que, na maioria dos países europeus, a promoção da saúde não consta dos objetivos do planeamento dos transportes, embora desde o final da década de 1980, tenham surgido indicações sobre os benefícios de incluir a temática do transporte no movimento da saúde pública, com especial foco na sinistralidade rodoviária, poluição atmosférica e sonora.

O transporte e a mobilidade são fonte de problemas para a saúde. No entanto, algumas soluções de mobilidade podem contribuir para a melhoria das condições gerais de saúde. Segundo Perdu, 2006, podem ser identifacadas três tipos de relações entre o transporte e a mobilidade e as condições de saúde: 
1) o impacte negativo que o transporte motorizado tem na qualidade do ar e níveis de ruído, afetando a saúde das populações (maior propensão de doenças respiratórias, cardiovasculares, cancerígenas e stress);

2) o encorajamento ao recurso a formas ativas de transporte como a caminhada, a utilização da bicicleta ou do transporte público;

3) a segurança do sistema de transporte, reduzindo e evitando acidentes rodoviários (adaptado de PERDUE, 2006).

O transporte motorizado para fins pessoais ou comerciais é dado como um dos grandes responsáveis do consumo de combustível fóssil, reforçado pelo aumento das distâncias, crescente número de viagens motorizadas, e maiores e mais frequentes períodos de congestionamento (YOUNGER et al., 2008). Sublinha-se ainda o ruído proveniente do transporte como altamente prejudicial à saúde, afetando a capacidade de comunicação, o rendimento escolar e laboral, o sono e a capacidade auditiva, com impacte negativo nos problemas cardíacos e hipertensão (WHO-EUROPE, 1999).

Já a deslocação ativa promove benefícios físicos e psicológicos bem como fortalece as redes sociais locais. As deslocações locais não motorizadas apresentam uma relação positiva com a saúde, havendo estudos que demonstram que os indivíduos que realizam deslocações utilitárias a pé ou de bicicleta apresentam menor Índice de Massa Corporal (BROWN et al, 2008). As deslocações ativas dependem não só das infraestruturas de transporte, mas também da qualidade, segurança e da disponibilidade de comércio, serviços, equipamentos e infraestruturas na proximidade (SAELENS et al., 2003; FREUDENBERG et al., 2011; MARQUES DA COSTA et al, 2011). Neste contexto, determinadas características do ambiente construído promovem deslocações pedonais, de bicicleta ou de transporte coletivo, reduzem as distâncias percorridas e reforçam a segurança dos trajetos. Tais intervenções potenciam impactes positivos na qualidade do ar, no aumento da atividade física ou no reforço do capital social (WHO-EUROPE, 1999). Consequentemente, promove-se a redução de incidência de doenças respiratórias, cardiovasculares e cancerígenas, bem como a promoção da saúde mental, diminuindo a propensão para a depressão e ansiedade. Destaca-se ainda a utilização do transporte coletivo, associada à redução da utilização do automóvel, evitando o congestionamento e emissões de 
poluentes; e simultaneamente à promoção da atividade física, com o acesso ao transporte que se realiza a pé ou de bicicleta (FREUNDEBERG et al., 2011).

Também a sinistralidade, em particular a rodoviária, é um problema grave para a saúde pública com impacte tanto na taxa de mortalidade como na incapacidade temporária ou definitiva dos indivíduos resultante dos acidentes. A sinistralidade rodoviária é influenciada não apenas pelas condições do ambiente urbano ou pelas condições atmosféricas, mas também por fatores comportamentais dos condutores como a velocidade, o consumo de álcool, ou o incumprimento de outras normas de segurança por parte de condutores e de peões (WHO-EUROPE, 1999).

\section{ENQUADRAMENTO DA ÁREA DE ESTUDO}

A Área Metropolitana de Lisboa, em Portugal, é composta por 18 municípios, repartidos por duas NUTS III ${ }^{1}$ - Grande Lisboa e Península de Setúbal (Figura 1), com uma área total de 3.015 quilómetros quadrados, onde residem 2.821.876 habitantes, ou seja, 19\% da população nacional reside em apenas 3\% do território (INE-Censos, 2011).

(1)

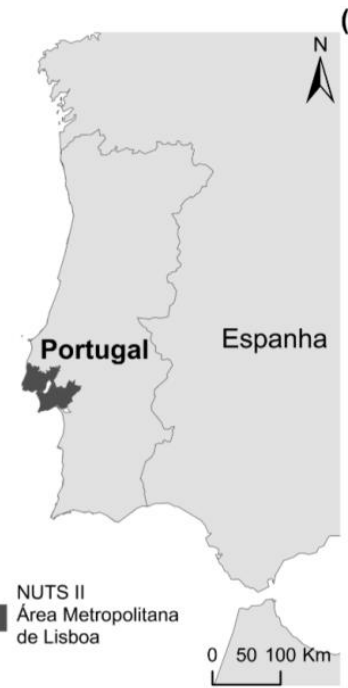

(2)

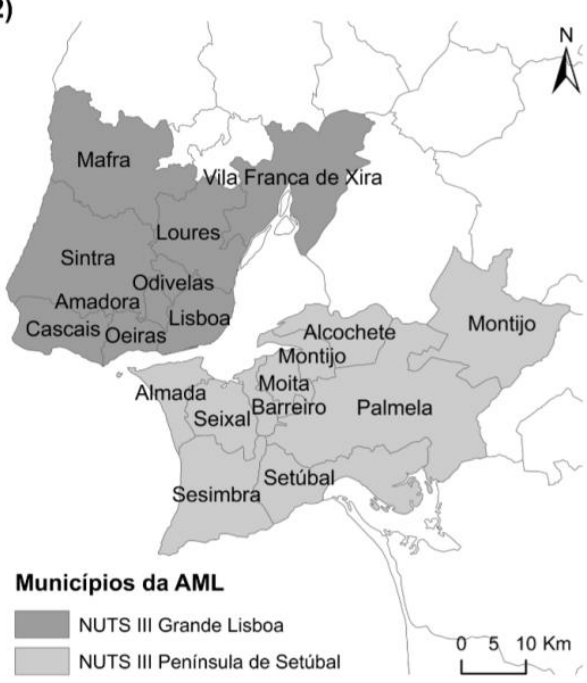

Figura 1. Área de estudo: (1) Área Metropolitana de Lisboa; (2) Municípios da AML por NUTS III. Fonte: Elaboração própria (2017).

\footnotetext{
${ }^{1}$ NUTS - Nomenclatura das unidades territoriais para fins estatísticos.
} 
O modelo urbano da AML apresentado no PROT-AML destaca Lisboa como o centro metropolitano, a par do eixo de centralidade Almada-Seixal-Barreiro. Segue-se um primeiro anel que reforça a acessibilidade entre as duas margens do Tejo, onde se localizam grandes polos de equipamentos e serviços, investigação e desenvolvimento: Amadora, Odivelas, Oeiras, Loures, Montijo e Moita. O segundo anel inclui os municípios de Cascais, Sintra e Setúbal, onde se localizam importantes áreas industriais, de armazenagem e logística. Numa posição periférica, localizam-se alguns polos turísticos, agrícolas e de preservação ambiental nos municípios de Alcochete, Sesimbra e Mafra (CCDR-LVT, 2010).

Os vários municípios metropolitanos apresentam características distintas. Lisboa é o único município que ultrapassa os 500.000 residentes (547.733 residentes), enquanto são sete os municípios com menos de 100.000 residentes, sendo Alcochete o de menor dimensão (17.569 residentes) (INE-Censos 2011), o que se traduz em valores de densidade populacional diferentes. Em 2011, a população infantil e juvenil (até aos 14 anos) da AML era de 15,5\% enquanto 18,2\% da população tinha idade superior a 65 anos. Se o município de Lisboa $(12,9 \%)$ apresentava a menor proporção de jovens, o contrário verifica-se em Alcochete (19,0\%). Também o caso de Lisboa (23,9\%) apresenta uma maior proporção de população idosa, por oposição a Sintra (13,7\%). A taxa de atividade na AML em 2011, ou seja, a proporção de população ativa em relação à população com 15 ou mais anos, era de 49,8\%, situando-se entre os valores da Amadora (47,5\%) e Lisboa (47,5\%), e Vila Franca de Xira (54,1\%), enquanto a taxa de desemprego da AML era de 12,9\% em 2011, variando entre os municípios de Mafra (9,1\%) e Moita (17,9\%).

\section{METODOLOGIA}

Tendo o caso português como exemplo, pretendeu-se identificar um sistema de indicadores que permita avaliar o contributo do "transporte e mobilidade urbana" para a cidade saudável, respondendo a três sub-domínios: 1) Transporte e mobilidade, 2) Saúde pública e civismo, e 3) Saúde pública e ambiente. 
No sub-domínio Transporte e mobilidade destacam-se aspetos associados aos movimentos pendulares, pela sua importância e regularidade; o destino, o modo e o tempo despendido. Num município saudável prevê-se que se privilegiem as deslocações não-motorizadas ou com recurso ao transporte coletivo, e de duração reduzida possibilitando a transferência do tempo poupado nas deslocações quotidianas para a realização de outras atividades, contribuindo para uma melhor qualidade de vida (EDWARDS \& TSOUROS, 2008; GIZ, 2011; RTPI, 2014; UIPT, 2016). Já o destino das deslocações pendulares reflete, em parte, a capacidade do tecido económico de cada município em reter os seus residentes ou em atrair não residentes (LYONS \& CHATTERJEE, 2008; EWING et al, 2011). A compra de automóveis novos representa o interesse no transporte individual como primeira opção de transporte, embora os novos veículos estejam tecnologicamente mais adaptados aos regulamentos ambientais (VAN MIERLO et al, 2004). Já a proximidade à rede de transporte coletivo revela a capacidade de o sistema de transportes públicos servir a população, sendo o acesso ao sistema uma condição fundamental para a sua utilização (GIZ, 2011; FASKUNGER, 2011).

No sub-domínio Saúde pública e civismo salientam-se as questões da sinistralidade rodoviária (GIZ, 2011; RTPI, 2014) bem como o da criminalidade associada à condução (por exemplo, a criminalidade associada à condução de veículo com taxa de álcool igual ou superior a 1,2 gramas por litro, limite aplicado em Portugal, ou a condução sem habilitação legal) (MASKALYK, 2003; FERREIRA, 2015).

No sub-domínio Saúde pública e ambiente destaca-se a qualidade do ar, considerando os limites legais de proteção da saúde humana (GIZ, 2011; RTPI, 2014), bem como o consumo de combustível por habitante, ou seja, a intensidade de uso de combustíveis fósseis, influenciado pelas opções modais, pelas características dos veículos e pelo estilo de condução (VAN MIERLO et al, 2004).

A partir desta leitura, construiu-se o sistema de indicadores presente no Quadro 1, que será alvo de uma análise quantitativa, primeiramente de forma a compreender a situação atual dos municípios da AML, e posteriormente identificando as principais desigualdades entre municípios através de medidas de dispersão (variância $\left(\mathrm{s}^{2}\right)$, desvio-padrão (s) e coeficiente de variação (CV)). 
Neste sistema ficam em falta indicadores associados à procura de bens, equipamentos, serviços e espaços verdes (GIZ, 2011), informação que não é recolhida de forma sistemática pelo sistema estatístico, apenas obtido por inquérito à população, normalmente associados a trabalhos de investigação (cf. MARQUES DA COSTA (2011); LOURO (2011); MARQUES DA COSTA et al. (2011) para casos da AML, Portugal).

A identificação do impacte da cada um dos indicadores do domínio dos transportes para o desenvolvimento de territórios saudáveis teve em atenção não só a bibliografia referenciada, como 0 relatório seminal publicada em 1999 em que se procurava identificar os indicadores que permitissem integrar os transportes e o ambiente a partir da informação estatística portuguesa disponível (PORTUGAL, 1999).

\begin{tabular}{|c|c|c|c|}
\hline \multirow{2}{*}{ Domínio } & Código & \multirow{2}{*}{ Indicador } & Impacte \\
\hline & Indic. & & Maior o valor... \\
\hline \multirow{5}{*}{ 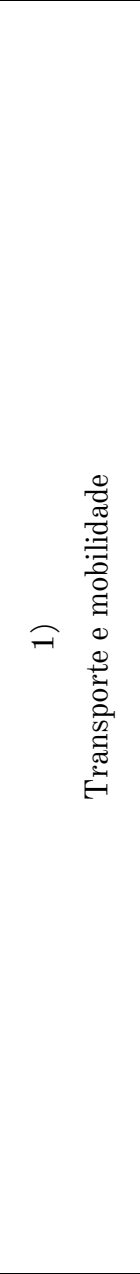 } & $\begin{array}{l}{[1.1]} \\
{[1.2]} \\
{[1.3]} \\
{[1.4]}\end{array}$ & $\begin{array}{l}\text { Destinos dos movimentos pendulares (INE, 2011) } \\
\text {. Proporção de deslocações pendulares por tipologia de destino } \\
\text {.. Dentro da freguesia de residência } \\
\text {.. Noutra freguesia do município de residência } \\
\text {.. Noutro município } \\
\text {. Proporção da população residente que entra na unidade territorial }\end{array}$ & $\begin{array}{c}\uparrow \\
\uparrow \downarrow \\
\uparrow \downarrow \\
\uparrow \downarrow\end{array}$ \\
\hline & $\begin{array}{l}{[2.1]} \\
{[2.2]} \\
{[2.3]} \\
{[2.4]} \\
{[2.5]}\end{array}$ & $\begin{array}{l}\text { Modo de transporte para movimentos pendulares (INE, 2011) } \\
\text { Proporção da população que utiliza modo de transporte individual } \\
\text {. Proporção da população que utiliza modo de transporte coletivo } \\
\text {. Proporção da população que utiliza mais do que um meio de transporte } \\
\text {. Proporção da população que se desloca a pé como meio de transporte } \\
\text {. Proporção da população que utiliza bicicleta como meio de transporte }\end{array}$ & $\begin{array}{l}\uparrow \downarrow \\
\uparrow \\
\uparrow \downarrow \\
\uparrow \\
\uparrow\end{array}$ \\
\hline & $\begin{array}{l}{[3.1]} \\
{[3.2]} \\
{[3.3]} \\
{[3.4]} \\
{[3.5]}\end{array}$ & $\begin{array}{l}\text { Duração dos movimentos pendulares (INE, 2011) } \\
\text {. Duração média dos movimentos pendulares } \\
\text {. Duração média dos movimentos pendulares por transporte individual } \\
\text {. Duração média dos movimentos pendulares por transporte coletivo } \\
\text {. Proporção dos movimentos pendulares até } 15 \text { minutos } \\
\text {. Proporção dos movimentos pendulares superiores a } 60 \text { minutos }\end{array}$ & $\begin{array}{l}\downarrow \\
\downarrow \\
\downarrow \\
\uparrow \\
\downarrow\end{array}$ \\
\hline & {$[4.1]$} & $\begin{array}{l}\text { Veículos ligeiros de passageiros novos vendidos por } 1000 \text { habitantes (ACAP/ } \\
\text { INE, 2013-15) }\end{array}$ & $\uparrow \downarrow$ \\
\hline & $\begin{array}{l}{[5.1]} \\
{[5.2]} \\
{[5.3]}\end{array}$ & $\begin{array}{l}\text { Proximidade à rede de transporte público (IMT/Elab. própria, 2017) } \\
\text {. Proporção de população residente até } 500 \text { metros de estações de comboio } \\
\text {. Proporção de população residente até } 500 \text { metros de estações de metro } \\
\text {. Proporção de população residente até } 500 \text { metros de paragens de autocarro }\end{array}$ & $\begin{array}{l}\uparrow \\
\uparrow \\
\uparrow\end{array}$ \\
\hline
\end{tabular}




\begin{tabular}{|c|c|c|c|c|}
\hline \multirow{2}{*}{$\overparen{\sim}$} & \multirow{2}{*}{ 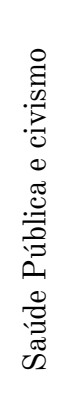 } & $\begin{array}{l}{[6.1]} \\
{[6.2]} \\
{[6.3]}\end{array}$ & $\begin{array}{l}\text { Sinistralidade rodoviária (ANSR/INE, 2014) } \\
\text {. Número de vítimas de acidentes de viação por } 1000 \text { habitantes } \\
\text {. Índice de gravidade dos acidentes de viação com vítimas } \\
\text {. Proporção de vítimas mortais em acidentes de viação }\end{array}$ & $\begin{array}{l}\downarrow \\
\downarrow \\
\downarrow\end{array}$ \\
\hline & & $\begin{array}{l}{[6.4]} \\
{[6.5]}\end{array}$ & $\begin{array}{l}\text { Taxa de criminalidade na área da condução (DGPJ/INE, 2015) } \\
\text {. Taxa de criminalidade por condução de veículo com taxa de álcool igual ou } \\
\text { superior a } 1,2 \mathrm{~g} / \mathrm{l} \\
\text {. Taxa de criminalidade por condução sem habilitação legal }\end{array}$ & $\downarrow$ \\
\hline \multirow[t]{2}{*}{$\widehat{\infty}$} & \multirow[t]{2}{*}{ 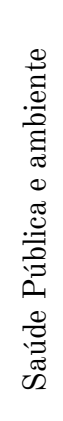 } & $\begin{array}{l}{[7.1]} \\
{[7.2]} \\
{[7.3]} \\
{[7.4]} \\
{[7.5]}\end{array}$ & $\begin{array}{l}\text { Qualidade do ar (APA, 2015) } \\
\text {. Percentagem do valor legal anual } \\
\text {.. Benzeno }(\mathrm{C} 6 \mathrm{H} 6) \\
\text {.. Óxido de Azoto }(\mathrm{NOx}(\mathrm{NO} 2)) \\
\text {.. Partículas poluentes } \mathrm{PM}_{2,5} \\
\text {.. Partículas poluentes } \mathrm{PM}_{10} \\
\text {.. Partículas poluentes } \mathrm{PM}_{10} \mathrm{EN}\end{array}$ & $\begin{array}{l}\downarrow \\
\downarrow \\
\downarrow \\
\downarrow \\
\downarrow\end{array}$ \\
\hline & & [7.6] & . Consumo de combustível automóvel por habitante (DGEG/INE, 2011-2015) & $\downarrow$ \\
\hline
\end{tabular}

Quadro 1. Indicadores do domínio transporte e mobilidade para territórios saudáveis. Avaliação do impacte: $\uparrow$ )

positivo; $\downarrow)$ negativo; $\downarrow$ ) simultaneamente positivo e negativo. Fonte: elaborado a partir de INE-Censos

2011; ACAP/INE; IMT; ANSR/INE; DGPJ/INE; APA; DGEG/INE.

\section{ANÁLISE DOS NDICADORES DE TRANSPORTE E MOBILIDADE URBANA PARA A CIDADE SAUDÁVEL NA ÁREA METROPOLITANA DE LISBOA}

Interessa então compreender a situação na $\mathrm{AML}$, relembrando que estes indicadores são fortemente influenciados pelas características da população e do sistema urbano metropolitano que refletem o padrão de localização da habitação, do emprego e das atividades económicas, gerando uma procura de transporte muito diferenciada. Todos os indicadores selecionados apresentam-se ao nível do município devido à disponibilidade de informação estatística (embora alguns estejam disponíveis a um nível inferior, o da freguesia), para maior utilidade na intervenção municipal da RPMS.

No sub-domínio Transporte e mobilidade, destacam-se indicadores associados ao destino, modo e duração dos movimentos pendulares, à posse de novos veículo automóvel e, ainda, à proximidade da população à rede de transporte público (Figura 2). 


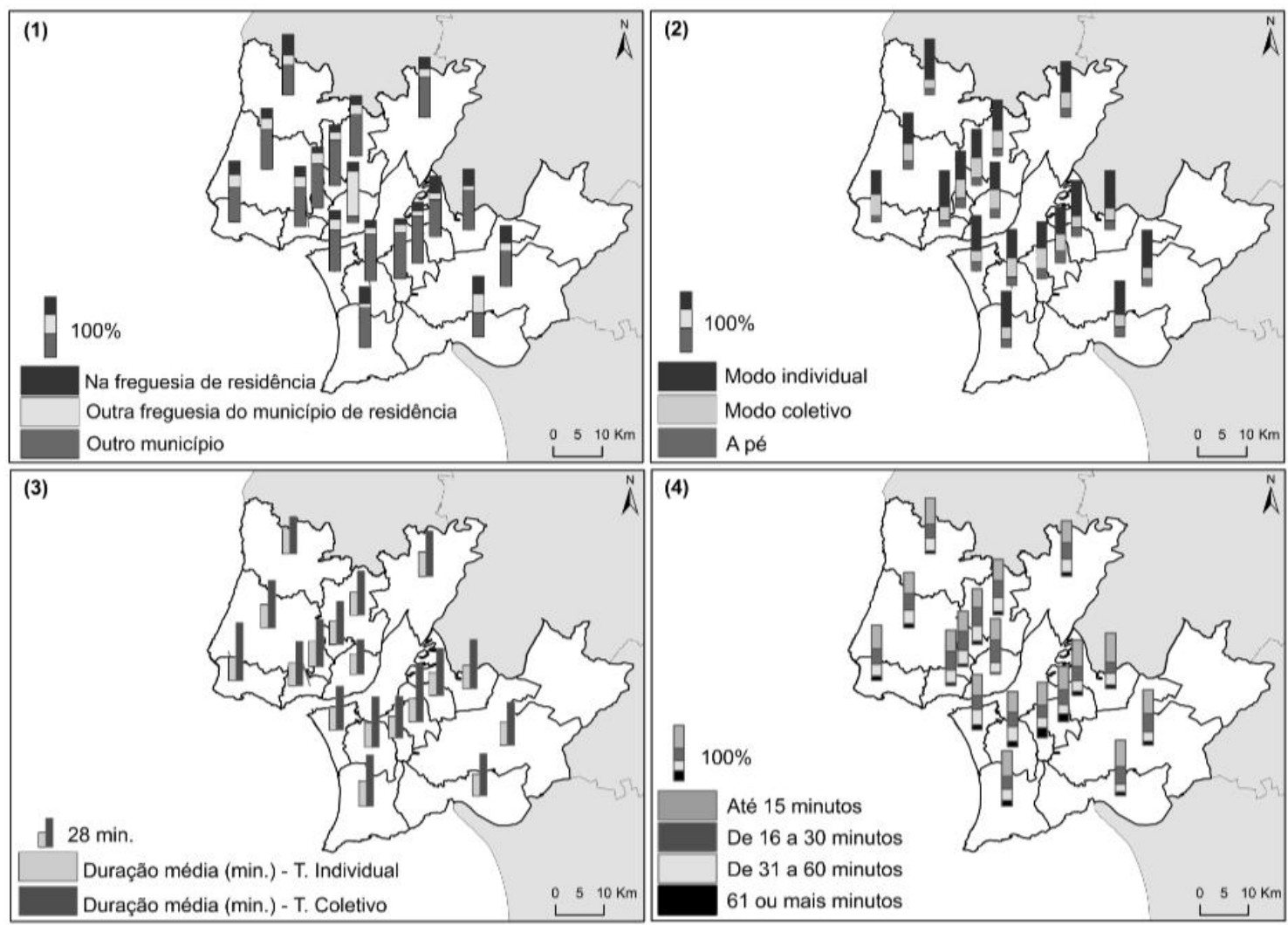

Figura 2. (1) Proporção de movimentos pendulares por destino; (2) Proporção de movimentos pendulares por modo de transporte; (3) Duração média dos movimentos pendulares; (4) Proporção dos movimentos pendulares de duração inferior a 15 minutos e superior a 60 minutos. Fonte: elaborado a partir de INE-Censos 2011.

Quanto ao destino das deslocações pendulares em 2011, na AML 30\% dos movimentos ocorriam dentro da freguesia de residência do indivíduo, 31\% destinava-se a outra freguesia do município de residência e os restantes 39\% dirigiam-se a outro município. Neste contexto, o município da Amadora (23\%) apresentava a menor proporção de movimentos pendulares dentro da freguesia de residência, contrariamente a Sesimbra (45\%). Quanto às deslocações pendulares para outras freguesias do município de residência, destaca-se Lisboa (63\%) por oposição a Alcochete (12\%). Finalmente, as proporções de movimentos para outro município revelam-se distintas: Lisboa (14\%) e Setúbal (25\%) num extremo contrastam com Amadora (54\%) e Odivelas (55\%).

Considera-se ainda a proporção da população residente que entra na unidade territorial, ou seja, o rácio entre a população residente que trabalha ou estuda em determinada unidade territorial residindo noutra unidade territorial e a população residente na primeira unidade territorial. Dentro da AML, os 
valores são discrepantes, sendo Sesimbra (6\%), Moita (7\%) e Seixal (7\%) os municípios com menor taxa de atração, contrariamente aos municípios de Oeiras (34\%) e, especialmente, Lisboa (78\%).

No que respeita aos modos de transporte utilizados para movimentos pendulares em $2011,55 \%$ da população empregada ou estudante da AML utilizava transporte individual, enquanto $29 \%$ utilizava transporte coletivo. Ainda no conjunto da AML, $24 \%$ da população empregada ou estudante necessitava de combinar diferentes modos de transporte. Quanto à utilização do transporte individual esta revelava-se bastante variada entre municípios: entre 43\% em Cascais e 47\% no Barreiro, e $73 \%$ em Mafra. Por sua vez, a proporção de utilização de transporte coletivo situa-se entre os $36 \%$ no Barreiro e em Odivelas, e os $16 \%$ em Mafra. A necessidade de combinar dois ou mais modos de transporte é também distinta, sendo mais evidente em Cascais (34\%) e no Seixal (30\%), e menos em Mafra (12\%) e Palmela (15\%). Ainda em 2011, a proporção de empregados e estudantes que se deslocava a pé como modo principal situava-se entre os 11\% em Cascais e Mafra, e os $21 \%$ na Moita. Por fim, a utilização da bicicleta era residual, situando-se entre os 0,1\% em Odivelas, Amadora, Loures, Oeiras e Sintra e 0,9\% no Montijo.

A duração média dos movimentos pendulares da população residente empregada ou estudante na AML em 2011 era de 26 minutos, situando-se entre os 23 minutos em Lisboa e Setúbal e os 33 minutos no Barreiro. Quando analisado por modo de transporte, verificam-se grandes diferenças, a começar pela média metropolitana: 22 minutos quando utilizado o transporte individual e 43 minutos utilizando o transporte coletivo. Enquanto o tempo médio de deslocação por transporte individual é muito semelhante entre os vários municípios, entre 20 minutos em Lisboa e 25 minutos na Almada, já utilizando o modo de transporte coletivo a duração é mais diferenciada, situada entre os 34 minutos em Lisboa e os 56 minutos no Barreiro. Surgem assim casos em que o diferencial entre a duração média das deslocações por modo coletivo e individual não chega a 15 minutos (casos de Mafra e Lisboa), enquanto noutros casos a diferença é superior a 30 minutos (casos da Moita e Barreiro).

No contexto da AML, a proporção de movimentos pendulares até 15 minutos era de 40\% em 2011. Apenas Alcochete apresentava mais de metade das deslocações pendulares com duração inferior a um 
quarto de hora (53\%). Por outro lado, o município de Odivelas apresentou a menor proporção (34\%), a par da Amadora (37\%). Já a proporção de movimentos pendulares superiores a 60 minutos em 2011 na AML era de 7\%. Neste contexto, assinala-se uma maior variação entre os municípios, sendo o Barreiro (18\%) e Moita (15\%) os que apresentavam os valores mais elevados, contrariamente a Lisboa (3\%), Amadora (5\%), Mafra (5\%) e Oeiras (5\%).

Considerando o triénio 2013-2015, 97\% dos veículos novos vendidos foram veículos ligeiros, sendo residual a aquisição de pesados de passageiros, pesados de mercadorias e tratores agrícolas. Relativamente aos veículos ligeiros, apenas dois casos apresentam valores acima da média metropolitana (65 veículos novos por 1000 habitantes): Lisboa (121 veículos novos por 1000 habitantes) e Oeiras (122 veículos novos por 1000 habitantes), municípios onde se verifica um volume populacional considerável associado a um elevado poder de compra, e também onde há maior concentração de sedes de empresas, responsáveis por uma parte muito significativa das aquisições de novos veículos.

Analisa-se ainda a proporção de população residente a menos de 500 metros (em distância euclidiana) das estações ou paragens de transporte público coletivo, considerando o comboio, o metropolitano e o autocarro. Tais resultados são condicionados pela configuração e densidade das redes de transporte (Fig. $3(1,2)$ ) e da distribuição da população residente. Para o conjunto da AML, é a rede de autocarros que apresenta uma maior cobertura de população até 500 metros das suas paragens (96\%), seguida da rede de metropolitano (13\%) (dada a sua localização concentrada em áreas de grande densidade populacional), e da rede de ferroviária (11\%) (localizada ao longo de quatro eixos na AML: linha de Sintra, Cascais e do Norte na margem norte e eixo Norte-Sul, ligando as duas margens) (Figura $3(3))$. 


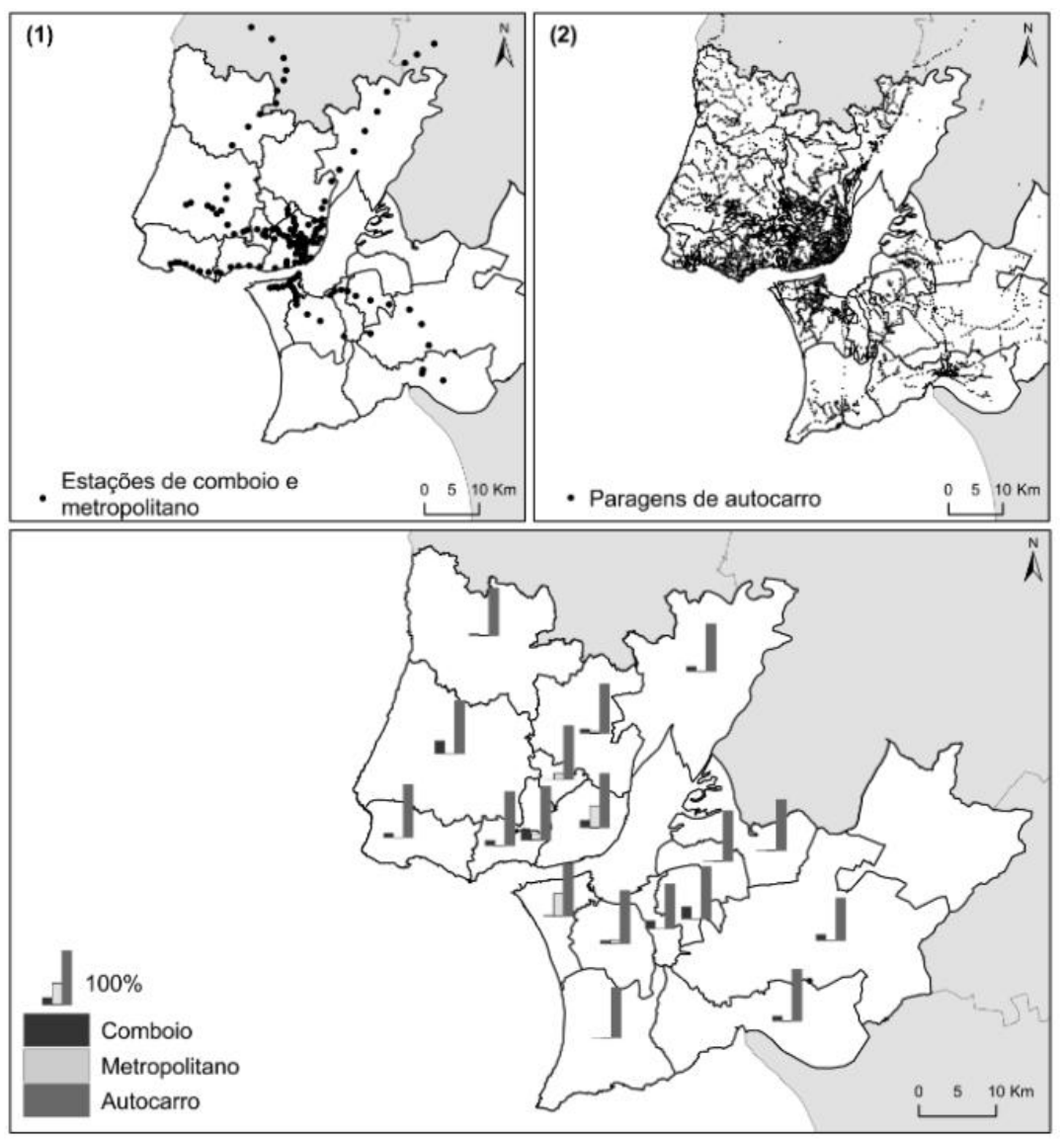

Figura 3. (1) Estações de comboio e metropolitano; (2) Paragens de autocarros; (3) Proporção de população residente a 500 metros de estações e/ou paragens por modo de transporte. Fonte: (1 e 2) elaborados a partir de IMT (2016), (3) elaboração própria (2017).

Quanto à rede de autocarros, todos os municípios apresentam elevadas taxas de cobertura. Destacamse Odivelas, Lisboa e Amadora que abrangem 100\% da sua população nas proximidades de pelo menos uma paragem. Apenas quatro municípios apresentam valores inferiores a 90\%: Palmela (79\%), Barreiro (83\%), Vila Franca de Xira (88\%) e Mafra (88\%), concelhos onde a dispersão populacional é mais significativa. No que respeita à rede de metropolitano pesado, esta circunscreve-se ao município de Lisboa (40\%) e a três concelhos limítrofes (Amadora (13\%), Odivelas (11\%) e Loures (4\%)). Já a rede de metropolitano ligeiro serve os municípios de Almada (42\%) e Seixal (7\%). Por fim, considerando a rede ferroviária pesada, quatro municípios não apresentam qualquer residente a menos de 500 metros das estações de comboio (Montijo, Sesimbra, Alcochete e Odivelas), sendo os casos da Amadora (19\%), Moita (23\%) e Sintra (24\%) os que apresentam maiores proporções deste indicador. 
No sub-domínio Saúde pública e civismo, enquadram-se indicadores de sinistralidade rodoviária e de criminalidade rodoviária. No contexto da sinistralidade rodoviária consideram-se o número de vítimas de acidentes de viação por 1000 habitantes e o índice de acidentes de viação com vítimas.
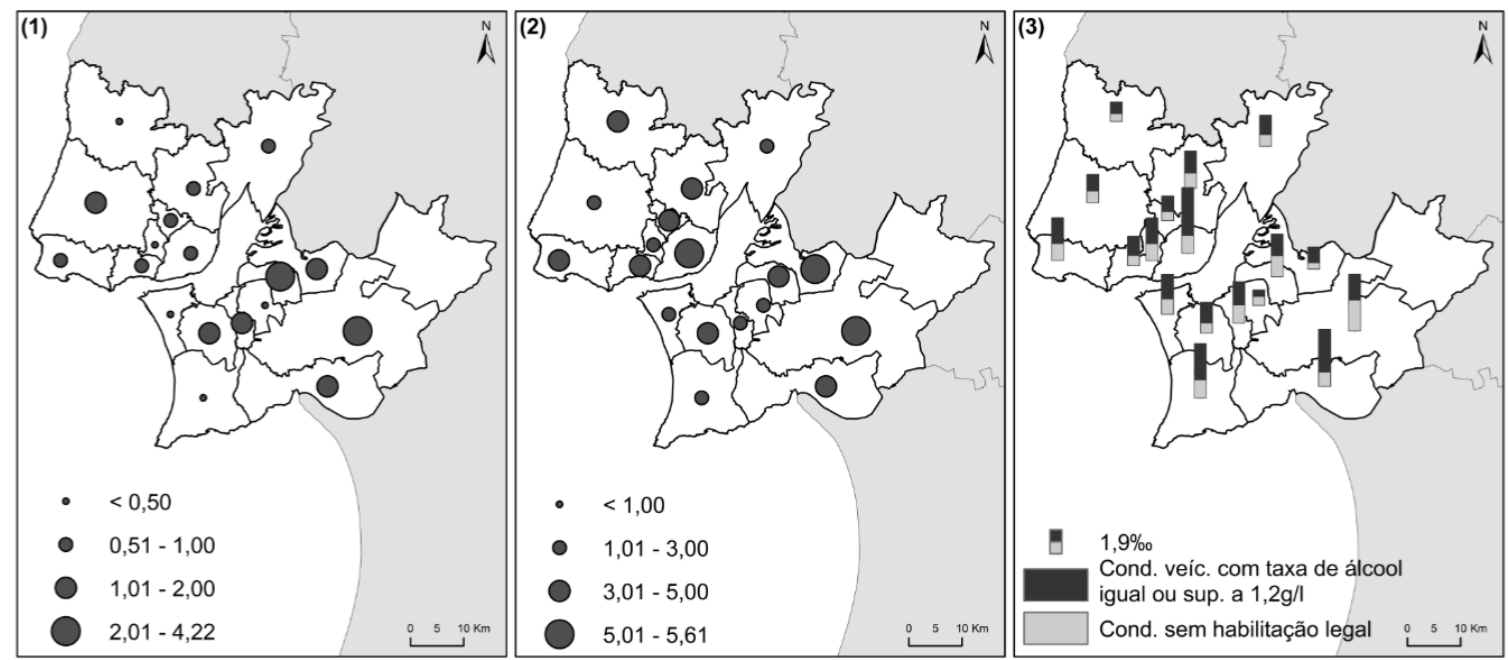

Figura 4. (1) Índice de gravidade dos acidentes de viação com vítimas; (2) Número de vítimas de acidentes de viação por 1000 habitantes; (3) Taxa de criminalidade por condução de veículo com taxa de álcool igual ou superior a 1,2g/l e Taxa de criminalidade por condução sem habilitação legal. Fonte: elaborado a partir de (1) e (2) - ANSR/INE (2014); (3) - DGPJ/INE (2015).

Em 2015, o índice de gravidade dos acidentes de viação com vítimas, ou seja, o número de óbitos por cada 100 acidentes de viação, era de 1,0 para a AML (Figura 4 (1)). Em três municípios o índice foi nulo (Amadora, Moita e Sesimbra), e em sete municípios o índice foi superior à média metropolitana, com destaque para Palmela $(4,1)$ e Montijo $(4,2)$. No mesmo período, registaram-se 3,8 vítimas de acidentes de viação por 1000 habitantes na AML, fenómeno mais patente em Alcochete $(5,6 \%$ e Lisboa $(5,6 \%)$, contrariamente aos casos de Sesimbra $(2,5 \%$ ) e Moita $(2,2 \%$ ) (Figura 4 (2)). Ainda em 2015, registaram-se $0,8 \%$ de vítimas mortais do total de vítimas contabilizadas na AML (entre 3\% no Montijo e 0,2\% em Almada,).

Quanto à taxa de criminalidade rodoviária (número de crimes rodoviários por cada 1000 residentes), destacam-se a condução de veículos com taxa de álcool igual ou superior a 1,2 grama por litro e a condução sem habilitação legal (Figura 4 (3)). Em 2015, a taxa de criminalidade por condução sem 
habilitação legal na AML era de 1,1\%o, variando entre os 0,5\%o em Alcochete e 2,4\%o em Palmela. Já a taxa de criminalidade por condução de veículo com taxa de álcool igual ou superior a 1,2 grama por litro era de 2,1\% na AML (entre 0,5\% na Moita e 3,7\% em Lisboa). Somando as duas tipologias destaca-se uma maior criminalidade rodoviária nos municípios de Lisboa $(5,1 \%$ ), Palmela (4,4\%o) e Setúbal $(4,4 \%)$.

Por fim, no sub-domínio Saúde pública e ambiente apresentam-se dois indicadores. Primeiramente analisam-se os Índices anuais da Qualidade do ar, monitorizados pela Comissão de Coordenação e Desenvolvimento Regional de Lisboa e Vale do Tejo (CCDR LVT, 2016). Tais índices têm por base os limiares da conformidade legal ${ }^{2}$ e são calculados por estação de controlo. Os Índices criados para 0 nível municipal foram calculados pela média aritmética dos Índices anuais da Qualidade do ar das várias estações de cada município. Os municípios que não possuem estações de medição não apresentam qualquer resultado.

A CCDR LVT (2016) apresentou a medição de cinco poluentes em 2015 (Figura $5(1,2)$ ):

1) óxido de azoto $\left(\mathrm{NO}_{\mathrm{x}}\right)$ : entre $17 \%$ em Palmela e $85 \%$ em Lisboa;

2) partículas poluentes $\mathrm{PM}_{2,5}$ : entre 47\% em Palmela e 54\% em Almada;

3) partículas poluentes $\mathrm{PM}_{10}$ : entre $42 \%$ em Oeiras e $76 \%$ em Cascais;

4) partículas poluentes $\mathrm{PM}_{10 \text { EN }}$ : entre $38 \%$ em Oeiras e $70 \%$ em Cascais;

5) benzeno $\left(\mathrm{C}_{6} \mathrm{H}_{6}\right)$ : apenas três municípios monitorizam este poluente, com índices de $11 \%$ em Setúbal, 14\% em Lisboa e 25\% em Cascais.

\footnotetext{
${ }^{2} \mathrm{O}$ índice Anual corresponde a uma percentagem do valor legal, calculado dividindo os resultados anuais obtidos em cada estação pelo valor legal definido para cada indicador (em percentagem), sendo que um índice de 100\% assume que o resultado anual do indicador é igual ao valor legal (CCDR LVT, 2016).
} 

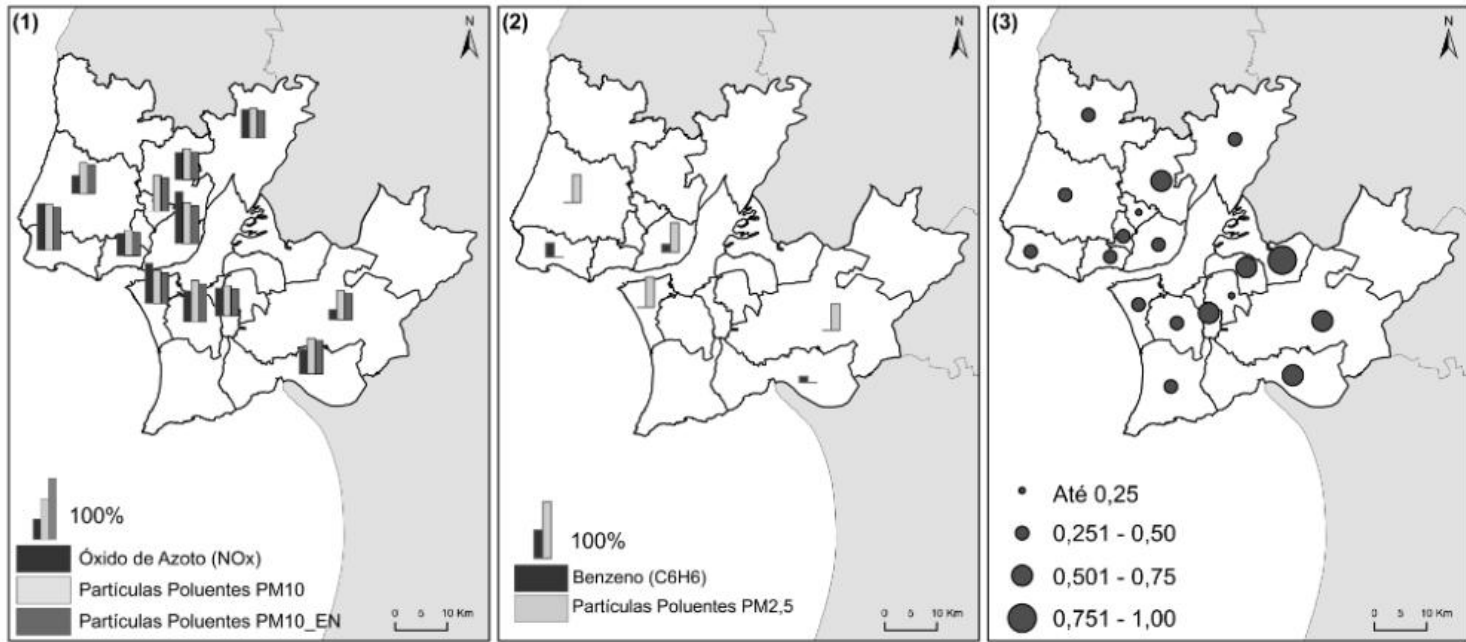

Figura 5. (1) Índice anual da qualidade do ar - $\mathrm{NO}_{\mathrm{x}}, \mathrm{PM}_{10}, \mathrm{PM}_{10} \mathrm{EN}$; (2) Índice anual da qualidade do ar - $\mathrm{C}_{6} \mathrm{H}_{6} \mathrm{e}$ $\mathrm{PM}_{2,5}$; (3) Consumo de combustível automóvel por habitante (tep/habitante). Fonte: (1) e (2) - APA (2015);

$$
\text { (3) - DGEG (2015). }
$$

Por último, o consumo de combustível automóvel por habitante (Fig. 5 (3)) era de 0,42 tep por habitante no conjunto da AML em 2015, estando onze municípios abaixo da média metropolitana, sobressaindo a Moita (0,21 tep/hab.) e Odivelas (0,22 tep/hab.), por oposição a Alcochete (0,95 tep/hab.) e Setúbal (0,73 tep/hab.).

Classificando os níveis de dispersão com base no coeficiente de variação (Tabela 1), dos 29 indicadores em estudo: 11 indicadores apresentam um CV Baixo ( $<\mathrm{CV}-1 \mathrm{~s}), 4$ indicadores apresentam um CV Médio (entre [CV-1s] e [CV+1s]), e 14 indicadores apresentam um CV Alto $(>\mathrm{CV}+1 \mathrm{~s})$.

\begin{tabular}{|c|c|c|c|c|c|c|c|c|c|c|}
\hline Indicador & Média & & nimo & Má & ximo & Ampli & Variância & $\begin{array}{c}\text { Desvio- } \\
\text { padrão (DP }\end{array}$ & Coefic. de & Class. \\
\hline [1.1] Na freguesia & 16,9 & 10,2 & Barreiro & 35,1 & Mafra & 24,9 & 66,2 & 8,1 & 48,1 & Alto \\
\hline [1.2] Outra freguesia & 27,4 & 6,8 & Sesimbra & 73,2 & Lisboa & 66,4 & 296,6 & 17,2 & 62,9 & Alto \\
\hline [1.3] Outro município & 55,7 & 11,1 & Lisboa & 76,8 & Seixal & 65,6 & 280,9 & 16,8 & 30,1 & Baixo \\
\hline [1.4] População que entra & 7,6 & 6,0 & Sesimbra & 77,7 & Lisboa & 71,7 & 366,3 & 19,1 & 252,3 & Alto \\
\hline [2.1] Transp. individual & 55,0 & 42,8 & Cascais & 72,4 & Mafra & 29,7 & 68,8 & 8,3 & 15,1 & Baixo \\
\hline [2.2] Transp. coletivo & 29,4 & 16,0 & Mafra & 37,6 & Cascais & 21,6 & 48,4 & 7,0 & 23,7 & Médio \\
\hline [2.3] Dois ou mais transp. & 23,7 & 11,8 & Mafra & 34,1 & Cascais & 22,3 & 31,9 & 5,6 & 23,8 & Médio \\
\hline [2.4] A pé & 15,3 & 11,2 & Cascais & 21,0 & Moita & 9,8 & 7,1 & 2,7 & 17,4 & Médio \\
\hline [2.5] Bicicleta & 0,2 & 0,1 & Odivelas & 0,9 & Montijo & 0,9 & 0,1 & 0,2 & 108,5 & Alto \\
\hline [3.1] Duração média & 26,4 & 23,0 & Lisboa & 32,9 & Cascais & 9,9 & 6,7 & 2,6 & 9,8 & Baixo \\
\hline [3.2] Dur. média T. indiv. & 22,1 & 20,0 & Lisboa & 24,6 & Amadora & 4,7 & 1,5 & 1,2 & 5,5 & Baixo \\
\hline [3.3] Dur. média T. coletiv. & 42,5 & 33,6 & Lisboa & 55,9 & Cascais & 22,4 & 33,5 & 5,8 & 13,6 & Baixo \\
\hline
\end{tabular}




\begin{tabular}{|l|r|r|r|r|r|r|r|r|r|r|}
\hline [3.4] Dur. Até 15 min. & 40,0 & 34,2 & Moita & 52,7 & Montijo & 18,5 & 26,7 & 5,2 & 12,9 & Baixo \\
\hline [3.5] Dur. mais 60 min. & 6,9 & 2,9 & Almada & 18,2 & Oeiras & 15,3 & 14,0 & 3,7 & 54,3 & Alto \\
\hline [4.1] Veíc.Novos/1000hab. & 65,0 & 21,4 & Moita & 122,4 & Oeiras & 101,1 & 1227,3 & 35,0 & 53,9 & Médio \\
\hline [5.1] Prox.Comboio(500m) & 11,4 & 0,0 & Vários (4) & 24,0 & Sintra & 24,0 & 62,2 & 7,9 & 69,1 & Alto \\
\hline [5.2] Prox. Metro (500m) & 12,5 & 0,0 & $\begin{array}{r}\text { Vários } \\
\text { (12) }\end{array}$ & 42,0 & Almada & 42,0 & 200,1 & 14,1 & 113,6 & Alto \\
\hline [5.3] Prox.Autocarro(500m) & 96,3 & 78,6 & Palmela & 100 & Amadora & 21,4 & 41,4 & 6,4 & 6,7 & Baixo \\
\hline [6.1] Vitimas / 1000 hab. & 3,8 & 2,2 & Moita & 5,6 & Lisboa & 3,4 & 1,1 & 1,0 & 27,2 & Alto \\
\hline [6.2] Índice de gravidade & 1,0 & 0,0 & Vários (3) & 4,2 & Montijo & 4,2 & 1,4 & 1,2 & 118,0 & Alto \\
\hline [6.3] Prop. Vítimas mortais & 0,8 & 0,0 & Vários & 3,3 & Montijo & 3,3 & 0,8 & 0,9 & 108,5 & Alto \\
\hline [6.4] Tx.Criminal - Álcool & 2,1 & 0,5 & Moita & 3,7 & Lisboa & 3,2 & 0,7 & 0,8 & 39,2 & Alto \\
\hline [6.5] Tx.Criminal - S/ carta & 1,1 & 0,5 & Alcochete & 2,4 & Palmela & 1,9 & 0,2 & 0,4 & 40,1 & Alto \\
\hline [7.1] Qualid. Ar [NOx] & 58,0 & 17,0 & Palmela & 85,0 & Lisboa & 68,0 & 439,3 & 21,0 & 36,1 & Baixo \\
\hline [7.2] Qualid. Ar [PM2,5] & 50,5 & 47,0 & Palmela & 54,0 & Almada & 7,0 & 7,3 & 2,7 & 5,3 & Baixo \\
\hline [7.3] Qualid. Ar [PM10] & 56,3 & 42,0 & Oeiras & 76,0 & Cascais & 34,0 & 89,9 & 9,5 & 16,9 & Baixo \\
\hline [7.4] Qualid. Ar PM10_EN] & 51,5 & 38,0 & Oeiras & 70,0 & Cascais & 32,0 & 81,9 & 9,1 & 17,6 & Baixo \\
\hline [7.5] Qualid. Ar [C6H6] & 16,7 & 11,0 & Setúbal & 25,0 & Cascais & 14,0 & 36,2 & 6,0 & 36,1 & Alto \\
\hline [7.6] Combustivel_hab & 0,4 & 0,2 & Moita & 0,9 & Alcochet & 0,7 & 0,0 & 0,2 & 43,8 & Alto \\
\hline
\end{tabular}

Tabela 1. Medidas de dispersão relativas ao sistema de indicadores em estudo. Fonte: Elaboração própria (2017).

Relativamente ao destino dos movimentos pendulares, três dos indicadores revelam um alto nível de desigualdade entre municípios (1.1. Proporção de deslocações pendulares dentro da freguesia de residência, 1.2. Proporção de deslocações pendulares para outra freguesia do município de residência, e 1.4. Proporção da população residente que entra na unidade territorial), contrariamente ao indicador 1.3. Proporção de deslocações pendulares para outro município, que apresenta um CV baixo.

No que respeita ao modo de transporte utilizado, três indicadores apresentam um nível de desigualdade médio (2.2. Proporção da população que utiliza modo de transporte coletivo, 2.3. Proporção da população que utiliza mais do que um meio de transporte e 2.4. Proporção da população que se desloca a pé), enquanto se verifica maior desigualdade no indicador 2.5. Proporção da população que utiliza bicicleta como meio de transporte, e uma menor desigualdade entre municípios no indicador a 2.1. Proporção da população que utiliza modo de transporte individual.

Abordando a duração dos movimentos pendulares, todos os indicadores apresentam um baixo CV com a exceção do indicador 3.5. Proporção dos movimentos pendulares superiores a 60 minutos, que 
apresenta um nível de desigualdade alto entre os vários municípios da AML. O indicador 4.1. Veículos ligeiros de passageiros novos vendidos por 1000 habitantes apresenta um CV de nível médio.

Quanto à proximidade à rede de transporte público, os indicadores 5.1. Proporção de população residente até 500 metros de estações de comboio e 5.2. Proporção de população residente até 500 metros de estações de metropolitano apresentam um nível de desigualdade alto, contrariando o indicador 5.3. Proporção de população residente até 500 metros de paragens de autocarro, com um nível de desigualdade baixo, ou seja, existe maior semelhança entre municípios.

No sub-domínio Saúde pública e civismo, todos os indicadores revelam um CV alto, ou seja, verificamse consideráveis desigualdades entre municípios, tanto os associados à sinistralidade rodoviária (6.1. Número de vítimas de acidentes de viação por 1000 habitantes, 6.2. Índice de gravidade dos acidentes de viação com vítimas e 6.3. Proporção de vítimas mortais em acidentes de viação), como relativos à criminalidade na área da condução (6.4. Taxa de criminalidade por condução de veículo com taxa de álcool igual ou superior a 1,2 grama por litro e 6.5. Taxa de criminalidade por condução sem habilitação legal).

Por fim, no sub-domínio Saúde pública e ambiente, a grande maioria dos indicadores relativos à qualidade do ar, para os municípios que dispõem de informação, apresentam um CV baixo (7.1. Óxido de Azoto $\left(\mathrm{NO}_{\mathrm{x}}\left(\mathrm{NO}_{2}\right)\right)$, 7.2. Partículas poluentes $\mathrm{PM}_{2,5}$, 7.3. Partículas poluentes $\mathrm{PM}_{10}$ e 7.4. Partículas poluentes $\mathrm{PM}_{10}$ EN $)$, com a exceção do 7.5. Benzeno $\left(\mathrm{C}_{6} \mathrm{H}_{6}\right)$ ) e do 7.6. Consumo de combustível automóvel por habitante, que apresentam um nível de desigualdade alto entre municípios da AML.

\section{CONSIDERAÇÕES FINAIS}

Este artigo enquadra-se na sugestão de Perdue (2005) de que a cidade saudável deve desenvolver uma visão multidisciplinar dos impactes na saúde, ampliando o conhecimento e dados disponíveis sobre a relação entre a saúde e, neste caso, o transporte e mobilidade urbana. Embora esta abordagem não 
seja recente, a operacionalização dos indicadores que permitem descrever e enquadrar as intervenções para a concretização da cidade saudável, enfrentam limitações que derivam do caráter multissetorial que a relação com o transporte e a mobilidade impõem.

No exercício metodológico aplicado à AML, verificou-se ser possível definir um sistema de indicadores que representam tal relação, através de três sub-domínios: 1) transporte e mobilidade, 2) saúde pública e civismo, e 3) saúde pública e ambiente. Destaca-se, contudo, a inexistência de alguns temas nos sistemas estatísticos nacionais, associados, por exemplo, ao potencial de acesso a equipamentos e serviços. A análise dos indicadores e das medidas de dispersão associadas permitiram ainda avaliar o nível de desigualdade de cada indicador considerando os vários municípios da AML através de medidas estatísticas simples.

Neste contexto, em 14 dos 29 indicadores identifica-se uma elevada desigualdade entre os municípios da AML, essencialmente associados aos destinos das deslocações pendulares, utilização da bicicleta, proporção de deslocações pendulares com duração superior a 60 minutos e com a proximidade dos residentes ao transporte público, com destaque para o comboio e o metropolitano, dentro do subdomínio do transporte e mobilidade; todos os indicadores associados à sinistralidade rodoviária e criminalidade no sub-domínio Saúde pública e civismo; e, por fim, ao consumo de combustível por habitante e à qualidade do ar quanto ao benzeno enquanto poluente atmosférico no sub-domínio Saúde pública e ambiente. Por sua vez, nos indicadores associados ao modo de transporte e duração das deslocações pendulares, aos vários índices de qualidade do ar e à proximidade dos residentes à rede de autocarro, verificou-se uma maior proximidade entre os municípios da AML.

Para uma análise mais sólida, é importante dar continuidade a este estudo considerando: outros períodos temporais, como forma de compreender as tendências de evolução dos indicadores, bem como o seu comportamento convergente ou divergente no contexto da AML; a aplicação de técnicas de análise multivariada, nomeadamente a análise de componentes principais, permitindo identificar os indicadores do sistema com maior e menor importância e a interação entre indicadores; e a replicação 
da metodologia para outras áreas metropolitanas ou regiões de Portugal para uma visão mais alargada, considerando os diversos modelos urbanos.

\section{REFERÊNCIAS}

ALVES, Lidiane A., COSTA, N. M., RIBEIRO FILHO, Vitor. Mobilidade e acessibilidade na construção de uma Uberlândia acessível e saudável. In: RIBEIRO SOARES, B. et al (Org.). Construindo cidades saudáveis - Utopias e práticas.

Uberlândia: Assis Editora, 2017.p. 544, p. 269-296 [Disponível em

https://www.researchgate.net/publication/318348272_Construindo_Cidades_Saudaveis_utopias_e_praticas. Acesso em: 20 junho 2017].

BANISTER, David. Unsustainable Transport: City transport in the new century. New York: Routledge - Taylor \& Francis Group, 2005. p. 292.

BROWN, Austin L.; KHATTAK, Asad J.; RODRIGUEZ, Daniel A.. Neighborhood types, travel and body mass: a study of new urbanism and suburban neighborhoods in the US. Urban Studies, Los Angeles, n. 45, p. 963-988, April 2008.

CCDR LVT. PROT-AML: Plano Regional de Ordenamento do Território da Área Metropolitana de Lisboa. Proposta técnica final, versão não aprovada. Lisboa: CCDR LVT, 2010. p. 368.

COSTA, N. M. da. Mobilidade e transportes em áreas urbanas: O caso da Área Metropolitana de Lisboa. Lisboa: CEG-UL, 2011. p. 626 [Disponível em

https://www.researchgate.net/publication/299411997_Mobildade_e_Transportes_em_Areas_Urbanas_O_Caso_da_Area_M etropolitana_de_Lisboa. Acesso em: 15 abril 2017].

CEnTRE FoR HeAlth PROTeCtion. Basic Principles of Healthy Cities: Health Determinants. Hong Kong: Centre for Health Protection - Government of the Hong Kong Special Administrative Region, 2009. 6p.

DOORIS, Mark. Healthy Cities and Local Agenda 21: the UK experience-challenges for the new millennium. Health Promotion International, Oxford, v. 14, n. 4, p. 365-375, Dezembro 1999.

EC. EU Transport in figures: Statistical pocketbook 2016. EU, Luxemburgo, 2016. p. 160.

EDWARDS, Peggy; TSOUROS, Agis D.. A healthy eity is an active eity: a physical activity planning guide. Copenhagen: WHO-EUROPE, 2008. 90p.

EWING, Reid; MEAKINS, Gail; BJARNSON, Grace; HILTON, Holly. Transportation and land use. IN: DANNENBERG, Andrew L.; FRUNKIN, Howard; JACKSON, Richard J. (Orgs). Making healthy places: Designing and building for health, wellbeing and sustainability. Cidade: Island Press, 2011. 417p., p. 149-169.

FASKUNGER, Johan. Promoting Active Living in Healthy Cities of Europe. Journal of Urban Health: Bulletin of the New York Academy of Medicine, New York, v. 90, n. 1, p. 142-153, October 2013.

FERREIRA, Manuel Jesus. Criminalidade Rodoviária: os crimes dos condutores. 131f. Tese (Mestre em Ciências Policiais) - Instituto Superior de Ciências Policiais e Segurança Interna, 2015.

FREUDENBERG, Nicolas; GALEA, Sandro; VLAHOV, David. Cities and the health of the public. Nashville: Vanderbilt University Press, 2011. p. 374.

GIZ. Urban Transport and Health. Eschborn: GIZ/BMZ, 2011. 60p. 
HANCOCK, Trevor; DUHL, Leonard J.. Promoting Health in the Urban Context. WHO Healthy Cities papers, n. 1. Copenhagen: FADL Publishers, 1988. p. 54.

LOURO, Ana. 0 uso do tempo associado à mobilidade das famílias como elemento diferenciador na configuração de uma Comunidade Sustentável. Lisboa: CEG-UL, 2011 [Disponível em:

https://www.researchgate.net/publication/264165977_O_uso_do_tempo_associado_a_Mobilidade_das_familias_como_elem ento_diferenciador_na_configuracao_de_uma_comunidade_sustentavel. Acesso em: 15 abril 2017].

LOURO, Ana.; COSTA, N. M. da e MARQUES DA COSTA, Eduarda. A cidade saudável no planeamento municipal: exemplos de atuação na Área Metropolitana de Lisboa, Portugal. In: RIBEIRO SOARES, B. et al (Org.). Construindo cidades saudáveis - Utopias e práticas. Uberlândia: Assis Editora, 2017. p. 544, p. 34-71 [Disponível em https://www.researchgate.net/publication/318348272_Construindo_Cidades_Saudaveis_utopias_e_praticas. Acesso em: 20 junho 2017].

LYONS, Glenn; CHATTERJEE, Kiron. A human perspective on the daily commute: costs, benefits and trade-offs. Transport reviews, v. 28, n. 2, p. 181-198, February 2008.

MARQUES DA COSTA, Eduarda; LOURO, Ana; FUMEGA, João. Indicadores para o planeamento à escala local. Uma abordagem em torno do conceito de comunidade sustentável aplicado à Área Metropolitana de Lisboa. Lisboa: CEG-UL, 2011. p. 317 [Disponível em https://www.researchgate.net/publication/317006246_Indicadores_para_o_planeamento_a_escala_local_-

_Uma_abordagem_em_torno_do_conceito_de_Comunidade_Sustentavel_aplicado_a_Area_Metropolitana_de_Lisboa. Acesso em: 15 abril 2017].

MARQUES DA COSTA, Eduarda. Cidades saudáveis, saúde e sustentabilidade: dos conceitos às orientações de política na União Europeia. In: LIMA, Samuel do Carmo; MARQUES DA COSTA, Eduarda (Orgs). Construindo Cidades Saudáveis. Uberlândia: Assis Editora, 2013. 288p., p.45-64. Disponível em

https://www.researchgate.net/publication/308795399_Cidades_saudaveis_saude_e_sustentabilidade_dos_conceitos_as_orie ntacoes_de_politica_na_Uniao_Europeia_Construindo_Cidades_Saudaveis_org_Samuel_Carmo_Lima_and_Eduarda_Marque s_da_Costa_Ed_AssisUberlandi?amp\%3B_sg=zAzqBhH96Q_BsdGtv6SSV9Hg2PGboEnr5G4jmxtVwNzoqGPUzfRWNE3N T0p7oV0FydqYnzB0EhaG0pFc. Acesso em: 15 abril 2017.

MARQUES DA COSTA, Nuno. Mobilidade e transportes em áreas urbanas: $\mathrm{O}$ caso da Área Metropolitana de Lisboa. Lisboa: CEG-UL, 2011. 626p.. Disponível em https://www.researchgate.net/publication/299411997_Mobildade_e_Transportes_em_Areas_Urbanas_O_Caso_da_Area_M etropolitana_de_Lisboa. Acesso em: 15 abril 2017.

MASKALYK, James. Drinking and driving. Canadian Medical Association, v. 168, n. 3, p. 313, February 2003.

MCNEILL, Lorna Haughton; KREYTER, Matthew W.; SUBRAMANIAN, S. V. Social environment and physical activity: a review of concepts and evidence. Social Science \& Medicine, Elsevier, v. 63, p. 1011-1022, Maio 2006.

MINDELL, Jennifer S.; COHEN, Judith M.; WATKINS, Stephen; TYLER, Nicholas. Synergies between low-carbon and healthy transport policies. Proceedings of the Institution of Civil Engineers - Transport, London, v. 164, n. 3, p. 127-139. Agosto 2011.

PERDUE, Wendy Collins. Building Healthy Cities: Legal frameworks and considerations. In: GALEA, Sandro; RODRIGUE, Jean-Paul; COMTOIS, Claude; SLACK, Brian. The Geography of Transport Systems. Oxon: Routledge Taylor \& Francis Group, 2006. p. 284.

PORTUGAL, Ministério do Ambiente. Transportes e Ambiente. Indicadores de Integração. Lisboa; DGA, Diecção Geral do Ambiente, 1999. 111p. 
RODRIGUE, Jean-Paul, COMTOIS, Claude, SLACK, Brian. The Geography of Transport Systems. Oxon: Routledge Taylor \& Francis Group, 2006. 284p.

RTPI. Promoting Healthy Cities - Why planning is eritical to a healthy urban future. Planning Horizons, Glasgow, n. 3, p. 24-26, October 2014.

SAELENS, Brian, SALLIS, James F., FRANK, Lawrence D. Environmental correlates of walking and cycling: findings from the transportation, urban design, and planning literatures. Annals of Behavioral Medicine, Springer, v. 25, n. 2, p. 8091, Abril 2003.

TOLLEY, Rodney. Sustainable Transport. Cambridge: Woodhead Publishing, 2003. p. 740.

TORRES, Margarida; SILVA, Lígia T.; SANTOS, Luís; MENDES, José F. G.. Saúde e bem-estar em meio urbano: das políticas à prática. Revista Portuguesa de Saúde Pública, Lisboa, v. 31, p. 95-107, Janeiro/Junho 2013.

UITP. Policy Brief - Desvendando os benefícios da mobilidade para a Saúde. São Paulo: UITP, 2016. 4p.

UN. Human Development Report 2016: Human Development for Everyone, Nova Iorque: UNDP, 2016. p. 286.

VAN MIERLO, Joeri; MAGGETTO, G; VAN DE BURGWAL, E; GENSE, R. Driving style and traffic measures Influence on vehicle emissions and fuel consumption. Proceedings of the Institution of Mechanical Engineers, Part D, Journal of Automobile Engineering, vol. 218, 2004. p.1-8.

VLAHOV, David; GIBBLE, Emily; FREUDENBERG, Nicholas; GALEA, Sandro. Cities and Health: History, approaches, and key questions. Academic Medicine, v. 79, n. 12, p. 1133-1138, Dezembro 2004.

WHITEHEAD, Margaret; DAHLGREN, Göran. What can we do about inequalities in health?. Lancet, v. 338, p. 10591061, Outubro 1991.

WHO. Declaração de Jacarta sobre a Promoção da Saúde no Século XXI. Jacarta: WHO-Europe, 1997. p. 8.

WHO. What are social determinants of health?. Disponível em:

http://www.who.int/social_determinants/sdh_definition/en/. Acesso em: 22 Setembro 2016.

WHO-Europe (1). What is a healthy city?. Disponível em: http://www.euro.who.int/en/health-topics/environment-andhealth/urban-health/activities/healthy-cities/who-european-healthy-cities-network/what-is-a-healthy-city. Acesso em: 22 Setembro 2016.

WHO-Europe (2). Healthy Cities. Disponível em: http://www.euro.who.int/en/health-topics/environment-andhealth/urban-health/activities/healthy-cities. Acesso em: 22 Setembro 2016.

WHO-EUROPE. Healthy cities and the city planning process: A background document on links between health and urban planning. Copenhaga: WHO-Europe, 1999. p. 43.

WHO-EUROPE. Promoting physical activity and active living in urban environments: The role of local governments. Copenhaga: WHO-Europe, 2006. p. 66.

WILLIAMS, Lisa Marie. Between health and place understanding the built environment. Toronto: Wellesley Institute, 2013. p. 15.

YOUNGER, Margalit; MORROW-ALMEIDA, Heather R.; VINDIGNI, Stephen M.; DANNENBER, Andrew L. The built environment, climate change and health: Opportunities for Co-Benefits. American Journal of Preventive Medicine, Washington, v. 35, p. 517-526, November 2008. 